\title{
Percutaneous Neurolytic Celiac Plexus Block
}

\author{
Ashley M. Nitschke, MD ${ }^{1}$ Charles E. Ray, Jr., MD, PhD, FSIR ${ }^{1}$ \\ ${ }^{1}$ Department of Radiology, University of Colorado School of Medicine, \\ Aurora, Colorado \\ Semin Intervent Radiol 2013;30:318-321 \\ Address for correspondence Charles E. Ray Jr., MD, PhD, FSIR, \\ Department of Radiology, University of Colorado School of Medicine, \\ 12401 E. 17th Avenue, Aurora, CO 80045 \\ (e-mail: charles.ray@ucdenver.edu).
}

Percutaneous neurolytic celiac plexus block (PNCPB) is an excellent treatment option for patients with intractable abdominal pain due to upper abdominal malignancies or chronic pancreatitis. In these patients, chronic refractory pain significantly decreases quality of life and often requires high doses of narcotics, which can lead to serious adverse side effects. PNCPB has been shown to have long-lasting improvement in abdominal pain and decreased narcotic usage in 70 to $90 \%$ of patients. ${ }^{1}$ In addition, with fewer than $2 \%$ of patients experiencing major complications, PNCPB is a quick, safe procedure. $^{1}$

The celiac plexus is a network of ganglia that relay preganglionic sympathetic and parasympathetic efferent fibers and visceral sensory afferent fibers to the upper abdominal viscera. The visceral sensory afferent fibers transmit nociceptive impulses from the liver, gallbladder, pancreas, spleen, adrenal glands, kidneys, distal esophagus, and bowel to the level of the distal transverse colon. Located in the retroperitoneum just inferior to the celiac trunk and along the bilateral anterolateral aspects of the aorta, between the levels of T12-L1 disc space and L2, the celiac plexus can easily be reached by several different approaches. ${ }^{1,2}$ Most commonly, anterior or posterior approaches are chosen. In the anterior approach, a needle is inserted through the anterior abdominal wall directly into the region of the celiac plexus and neurolytic agent is injected into the antecrural space. Although this approach necessitates traversing abdominal structures including bowel and liver, this is generally inconsequential, well tolerated, and often quicker than other approaches ( - Fig. 1). Additionally, the anterior approach may be more comfortable for the patient, as they are placed in a supine position, compared with less comfortable prone or oblique positions used for posterior approaches. In the posterior approach, a needle is inserted through the paraspinous musculature into the region of the celiac plexus and neurolytic agent injected into the antecrural space. Other less common approaches include transaortic and trans-intervertebral disc.

At the authors' institution, PNCPB is performed nearly always by an anterior approach under computed tomographic (CT) guidance. CT guidance is a safe, popular choice that allows for excellent visualization of abdominal anatomy, preplanning, precise placement of needles, and observation of contrast/neurolytic agent diffusion. ${ }^{1}$ Alternatively, neurolysis of the celiac plexus can be performed under fluoroscopic or ultrasound guidance, as well as endoscopic ultrasound guidance. Fluoroscopic guidance allows better visualization of the region of interest than the original blind approach first performed nearly 100 years ago; however, poor resolution of surrounding structures including the stomach, pancreas, bowel, aorta, and spinal cord makes it a less frequently utilized technique. Ultrasound guidance allows for direct visualization of important vascular structures, particularly the aorta, celiac trunk, and superior mesenteric artery. Additionally, it is cheap, simple, and allows the user to observe diffusion of the neurolytic agent without using contrast. However, ultrasound guidance is user dependent and its usage may be limited depending on patient body habitus. More recently, endoscopic ultrasound-guided celiac plexus neurolysis has become an increasingly popular and safe alternative that potentially allows for direct visualization and targeting of the celiac ganglion.

\section{Technical Approach}

As outlined above, PNCPB can be performed in multiple ways; the following is the preferred approach typically used by the authors.

The patient is placed supine on the CT gantry, and a preliminary CT scan of the upper abdomen is performed without contrast. The initial scan is reviewed to confirm an adequate route to the celiac plexus region, and to confirm the lack of direct invasion of the celiac plexus by the underlying pathologic process. Although the procedure may still be performed in the setting of direct tumoral invasion of the plexus, the outcome is likely to be worse than in the setting of no direct invasion, and the patient should be so counseled. Unless invaded by tumor, the celiac plexus itself is rarely visualized on the initial CT scan.

An initial plan of using one or two needle approaches is made, and an appropriate skin site is chosen. A 21- to 22guage Chiba needle (Cook Medical, Inc., Bloomington, IN) is
Issue Theme Neurointerventions for the Interventional Radiologist; Guest Editors, Gregory M. Soares, MD, FSIR and Sun Ho Ahn, MD
Copyright @ 2013 by Thieme Medical Publishers, Inc., 333 Seventh Avenue, New York, NY 10001, USA. Tel: +1(212) 584-4662.
DOI http://dx.doi.org/ 10.1055/s-0033-1353485. ISSN 0739-9529. 


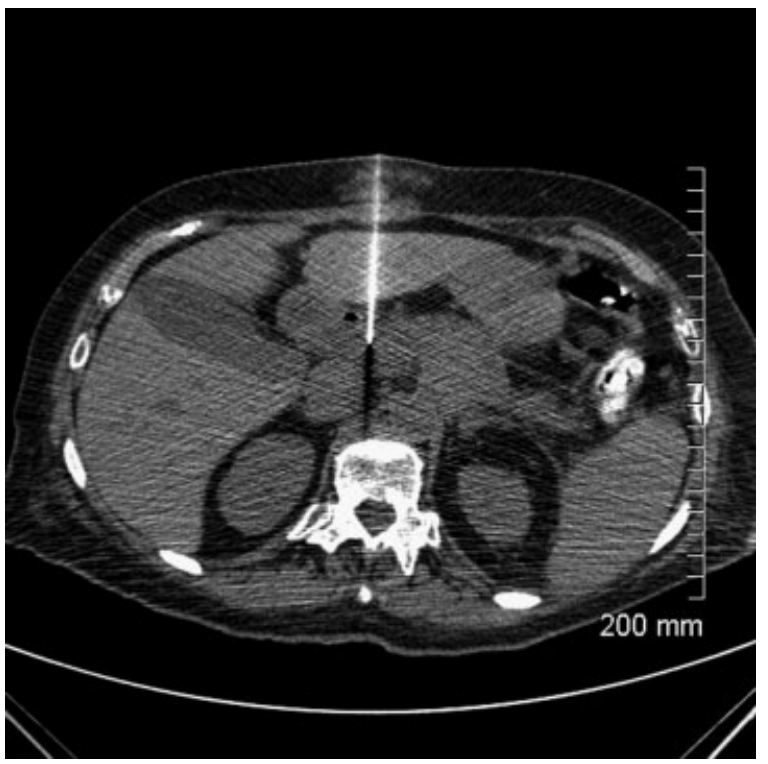

Figure 1 Axial noncontrast CT scan demonstrating anterior approach to PNCPB. Note the traversal of multiple abdominal organs; although disconcerting to the eye, this anterior approach very rarely causes any clinically significant injury to other abdominal organs.

advanced under intermittent $\mathrm{CT}$ guidance to the fat-containing space just dorsal and caudal to the celiac axis. Although disconcerting the first several times this procedure is performed, it is not at all uncommon to traverse liver, colon, bowel, pancreas, etc., on the way to the celiac plexus (although, it must be said, avoiding major blood vessels would be preferable!) (-Fig. 1). It is important to place the needle caudal, not cephalad, to the celiac axis; some operators (including the authors) have demonstrated improved outcomes when the injection is made closer to the superior mesenteric artery than the celiac axis. Once the needle is in position, an injection of 5 to $10 \mathrm{~mL}$ of dilute contrast $(1 \mathrm{~mL}$ contrast: $9 \mathrm{~mL}$ saline) is performed, and CT images obtained. Confirmation of contrast infiltrating around the celiac axis and lateral to the aorta is desired; if no such infiltration is noted, the needle should be repositioned in an attempt to maximize this distribution (-Fig. 2). Contrast visualized on both sides of the aorta is preferable but not mandatory; a lack of contrast bilaterally suggests that a second needle should be placed on the side without the contrast dispersion in order to maximize the celiac block.

Just prior to performing the block, the patient is asked what his or her pain level is at the moment. This baseline value, and the change imparted by the next step, is an important determinant on whether or not the needle is in the correct position. For this reason, the patient should be minimally sedated and have received little to no intravenous pain medications up to this point in the procedure. The authors prefer to perform a temporary block at this point in the procedure for two reasons. First, the temporary block can confirm adequate needle placement; if the patient goes from a pain level of, for instance, $5 / 10$ to $1 / 10$ following the temporary block, then the needle is likely in a very good position for the PNCPB. Second, a field block with local

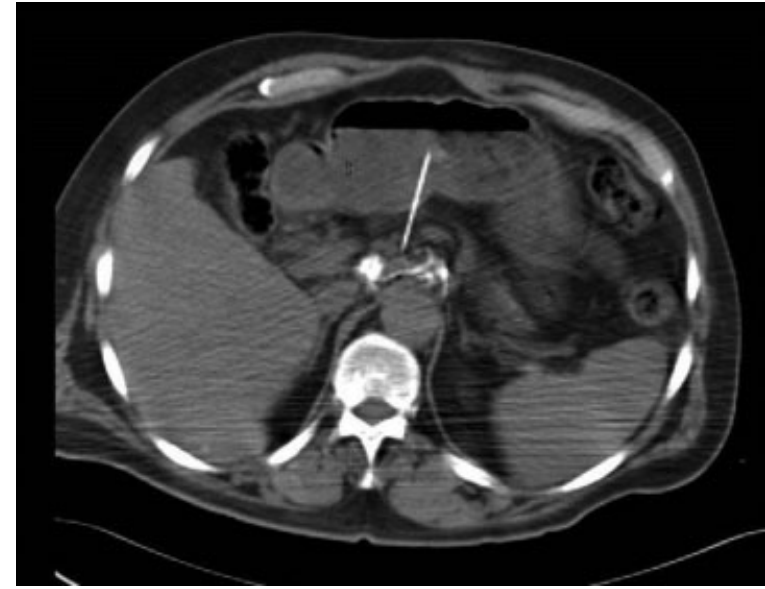

Figure 2 Axial CT scan following injection of dilute contrast around the celiac plexus. Note the dispersion of contrast around the aorta and mesenteric vessels.

anesthetic decreases the amount of pain patients may feel with the PNCPB, since the initial alcohol injection can cause significant, albeit brief, abdominal pain.

Once correct needle position is confirmed by contrast dispersion and improved pain with the temporary block, the patient is given a bolus of intravenous fentanyl and versed. Absolute alcohol is injected slowly (over 2 minutes) via the Chiba needle. Volume of the injectate varies, but typically is in the range of 10 to $40 \mathrm{~mL}$. The authors typically use $20 \mathrm{~mL}$ prior to repeating the CT scan. On the postprocedure CT scan, alcohol will appear black; further injection may be dictated depending on the results of the initial postprocedure $\mathrm{CT}$. The need for a second needle placement will also be determined by the postprocedure CT scan, based on distribution of the alcohol/dilute contrast. If a bilateral injection is needed, the procedure is repeated for the second needle exactly as it was performed for the first (- Fig. 3).

Following the postprocedure $\mathrm{CT}$, the patient is transferred to the postprocedural care area for a 1- to 2-hour recovery period. The patient is observed for signs of peritonitis and hypotension. If the patient does become hypotensive relative to their baseline blood pressure, orthostatic pressures are obtained prior to discharging the patient.

\section{Patient Selection}

In addition to the technical approach utilized, two of the most important aspects for a successful PNCPB are appropriate patient selection and communication. Basic preprocedural workup should include a physical exam, complete blood cell count, coagulation panel, and an abdominal CT to exclude relative contraindications to PNCPB including severe coagulopathy, thrombocytopenia, abdominal aortic aneurysm, local infection or sepsis, significant direct tumoral extension into the celiac plexus, or hypotension. Next, the extent of disease and origin of pain should be carefully evaluated to determine the likelihood of symptomatic relief by PNCPB. Patients with diffuse or multifocal disease are more likely to have poor 

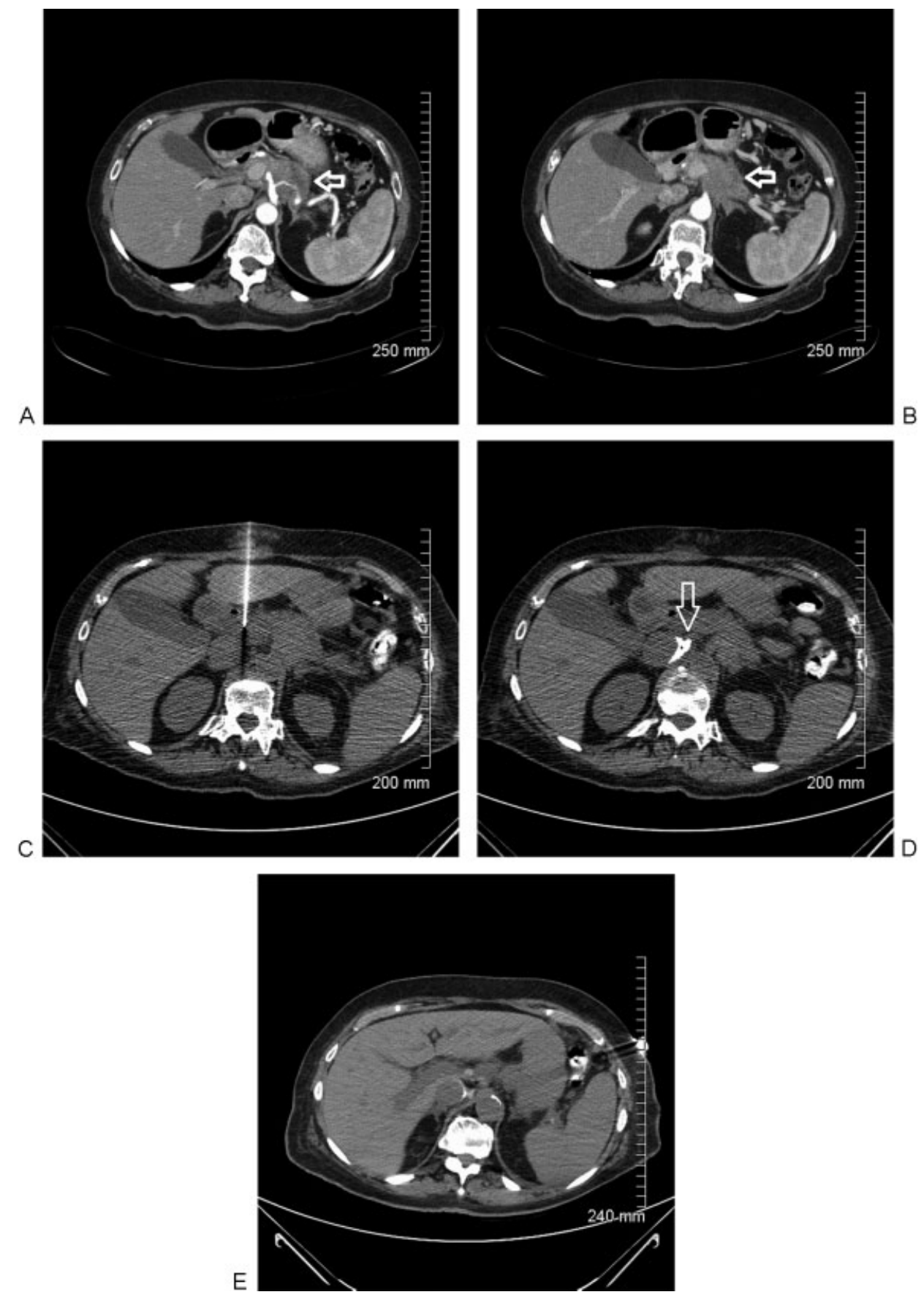

Figure 3 A 68-year-old woman with direct tumoral involvement of the left celiac plexus. (A and B) Diagnostic contrast-enhanced CT scan demonstrating direct infiltration of the celiac plexus region by tumor (arrows). Note the severe encasement of the splenic artery. As this is often a poor predictor of a good response, the patient was made aware of the likely limitations to the celiac block. (C) A right-sided approach was chosen, and a needle advanced to the region of the celiac plexus (same as Fig. 2). (D) Contrast diffusion (arrow) covers the anticipated region of the celiac plexus. A temporary block completely alleviated the patient's pain, so a permanent alcohol ablation was performed with $20 \mathrm{~mL}$ of absolute alcohol. (E) Postablation CTscan demonstrating contrast and alcohol diffusion (alcohol is black on the postablation CTscan). The patient was symptom free immediately following the ablation.

results, as some areas of involvement may be innervated by other pathways. ${ }^{1}$ Additionally, direct tumor invasion of the celiac plexus is a known predictor of poor outcome in PNCPB (-Fig. 3). For patients whose source of pain is in the descending or sigmoid colon, rectum, or other pelvic structures, celiac plexus block will be ineffective, since these areas are innervated by the hypogastric plexus; in such cases, percutane- ous hypogastric plexus neurolysis can be performed, which is also a safe and easy procedure with minimal side effects.

Once a patient has been selected as an appropriate candidate, it is important to thoroughly explain the steps of the procedure (particularly those involving patient participation), potential complications, and the expected outcome. Patients should be taught to hold their breath during needle 
advancement, which is crucial to avoid injury to adjacent structures. Another important step involving patient participation is assessing the level of pain during the procedure when the needle is in the expected location of the celiac plexus. Injection of local anesthetic at this point should result in decreased pain, confirming the proper position of the needle. If the patient reports no pain improvement, reposition of the needle may be necessary.

Patients should be warned of complications and side effects of the procedure, including temporarily increased abdominal, back, or shoulder pain that may be expected after the injection of the neurolytic agent. Finally, patients must understand that $100 \%$ pain relief may not be obtained, as most patients experience only partial relief of pain. However, some improvement in pain and quality of life, especially when combined with other pain treatment options, can be expected. When necessary, repeated PNCPB treatments may help reduce pain over time.

PNCPB is generally a safe procedure with rare serious complications. The most common complaint reported by nearly all patients during the procedure is severe abdominal or back pain that may radiate to the shoulder. This occurs due to the destruction of nerve fibers by the neurolytic agent and generally resolves within 72 hours. The next most common complications experienced after PNCPB include diarrhea and hypotension $(10-52 \%)^{2}$ This results from unopposed para- sympathetic stimulation caused by destruction of the sympathetic fibers within the celiac plexus. Postprocedural bed rest, monitoring of patients, and administration of intravenous fluids when necessary can relieve hypotension. Symptomatic treatment can be provided for diarrhea, which is usually self-limiting. Less frequent major complications have been reported in fewer than $2 \%$ of patients and include gastric or bowel perforation, vascular injury, hematoma, and chemical peritonitis. ${ }^{1}$ These complications may occur from direct penetration by a needle or by chemical inflammation from neurolytic agent, particularly if diffusion of the agent is not controlled. The most serious potential complication is paralysis of the lower extremities, which is extremely rare and reported to occur in fewer than $0.15 \%$ of patients. ${ }^{2}$

\section{Suggested Readings}

1 Kambadakone A, Thabet A, Gervais DA, Mueller PR, Arellano RS. CT-guided celiac plexus neurolysis: a review of anatomy, indications, technique, and tips for successful treatment. Radiographics 2011;31(6):1599-1621

2 Wang PJ, Shang MY, Qian Z, Shao CW, Wang JH, Zhao XH. CTguided percutaneous neurolytic celiac plexus block technique. Abdom Imaging 2006;31(6):710-718

3 Morello F. Percutaneous management of visceral pain. In: Ray CE $\mathrm{Jr}$, ed. Pain Management in Interventional Radiology. Cambridge, UK: Cambridge University Press; 2008:188-198 